\begin{tabular}{ccc}
\hline & International Journal of Engineering \& Technology, $7(2.7)(2018) 293-296$ \\
SPC & International Journal of Engineering \& Technology \\
Website: $w w w . s c i e n c e p u b c o . c o m / i n d e x . p h p / I J E T$ & Research Paper \\
\hline
\end{tabular}

\title{
Survey of privacy and security issues in IoT
}

\author{
A. Gopi ${ }^{1}$ *, M. Kameswara $\operatorname{Rao}^{2}$ \\ ${ }^{1}$ Research Scholar, KLEF, Vijayawada \\ ${ }^{2}$ Associate Professor, KLEF, Vijayawada \\ *Corresponding author E-mail: gopi.arepalli400@gmail.com
}

\begin{abstract}
The paper is a standard study of all the safety problems present inside the Internet of Thing (IOT) along with an study of the retreat problems to an give up-person can also face due to the reach of IOT. The greater part of the analysis is focused on the safety loop hole bobbing up not in of the statistics alternate technology utilized as a part of Internet of thing. No register measure to the security downsides has been examine in the article.
\end{abstract}

Keywords: Wireless Sensor Networks; IOT DOS; DDOS; Attacks; MFID

\section{Introduction}

Structure upon machine to Device (M2D)conversation learning of Bill [1], IOT encapsulates the origination of permitted stream of figures among the different inserted registering gadgets the utilization of the web as the sort of bury contact. The day and age "Web of Thing" changed in to first arranged by kevin ashton inside the yr 1110 [2]. With the reason exhibit propelled mode of verbal exchange between the numerous structures and plans as well like facilitate the interaction of human beings with the digital sur- roundings, IOT reveals its software in almost any discipline. But as with everything's the usage of the net infrastructure for records alternate, IOT to is vulnerable to diverse safety issues and has a few essential privations worries for the cease customers. Such IOT, in spite of all its modern skills in facts change place, a mistaken intention from the safety standpoint and right manner must be hold inside the preliminary section its own earlier than going for further improvement of IOT for an powerful and extensively prev- alent acceptance.

\section{Over view}

Computerized trade of statistics among organizations or two gadgets with none guide effort is the essential goal of the Internet of Things. This computerized statistics change among two gadgets takes location via some unique conversation tools, following are designated next section.

Remote Sensor Networks (RSN)

Marked in [3], RSN are structures of fair hubs such remote correspondence takes vicinity in excess of confined rate of recurrence and bandwidth. The interconnecting knobs of a distinctive wi-fi sensor network include the subsequent portions:
a) Sensing
b) Micro control
c) Recollection management
d) Frequency Transceiver range
e) Cell consumption

Because of the restricted correspondence scope of every sensor hub of a RSN, multi-hop hand-off of data take territory among the supply and the initial site. An essen- tial realities is amassed by method for the remote trans- vers through coordinated effort among the different hubs, that is move to the sink hub to centered directing nearer to the base station. The verbal exchange community shaped vigorously by using the use of wi-fi radio trans- ceivers allows data broadcast among nodes. Multi-hop broadcast of statistics needs specific nodes to take vari- ous visitors hundreds [2].

Frequency Or Medium Frequency Indicat- ed (F\&MFID)

In circumstances to the IOT, MFID innovation is uniquely used in data labels interrelating with others mechanically. MFID labels utilize radiofrequency waves for interfacing and trading information among each oth- er and not utilizing a necessity for design in the indistin- guishable line of vision or physical touch. It influences utilization of the remote time of Programmed Identifica- tion and Data To catch . A MFID is comprised of the accompanying added.

MFID labels.

In MFID label, a test is installed in microchip. The MFID label in like manner includes memory contrap- tions, which families a correct identifier suggested as EPC.MFID names are:

a) Updated label

This kind of name homes a power pack inside, it helps the correspondence and its supreme with its neighboring EPCs Ad-hoc from compelled sepa- ration.

b) Unconcerned label

In this sort of label, the records dispatch of EPC happens best by using its prompting by methods for a handset from pre-depicted collection of label. The loss of an inward battery in the reserved marks is traded by misuse of electromagnetic banner transmitted with the guide of a name book inductive paring as a supply of essentialness. (For bits of knowledge about usage of outside resources of quality an inactive label, per users can talk about with [4]).A MFID label actuates nearby a label per user, the EPC of the past being the making sense of moniker of an exact label underneath the examination of the end.

MFID per users 
The MFID per user includes as the accreditations radar of every identifier through its communication with an EPC of the label underits experiment .Effective certain- ties at the moment innovation in the back of MFID might be situated in [6].

\section{Sanctuary disputes and confidentiality anxi- eties}

Although the massive capacity of IOTs in the numerous compasses, the complete statement arrangement of the IOTs is defective commencing the sanctuary viewpoint and is inclined to damage of privateness for the cease consumers. Some of the utmost noticeable protection troubles afflicting the complete growing IOT device rise up available of the safety troubles gift inside the skills used in IOT for statistics convey from one device to an- other. Such as a number of the distinguished protection concerns reducing out from the barrier generations fol- lowing:

Safety troubles within the wi-fi sensing net- work (RSNs):

The classified courting of the innumerable safety prob- lems afflicting the sensor radar community is shown in Diagram 1.The unfair actions that can be accomplished in a wireless radar network may be classified beneath 3categories [7]:

a) Assaults on confidentiality and substantiation

b) Muted assaults on provision veracity

c) Outbreaks on community accessibility: The rejection of carrier (DoS) ([13], [13]) assault cascades below this class.

This avoidance of openness of data to valid users by methods for obscure $1 / 3$ festivity gatecrashers can take zone on distinct layers of a group [8 14], [15]:

Denali of Service assault in the manual lay- er:

The bodily stratum of an ad-hoc sensor network spreads out task choice and technology of provider incidence, modulated and demodulated, encrypted and decrypted, transmission and received statistics [13]. This level of their-fi transceiver network is criticized specially through. Overflow: In this kind of Denali of Service assault.

Denali of Service assault on the link layer: The connection layer of RSN multiplexes the various data stream, bears acknowledgment of information body, Medium accesses and missteps control. Similarly a hy- perlink layer ensures factor-factor or point many points consistency [14]. The Denial of service attacks occur- ring in this layers are:

a) Crash: This sort of DoS strike can be presented while hubs simultaneously exchange parcels of realities on the equivalent recurrence station. The accident of record parcels impacts in little modifications inside the bundle results in character of the parcel as a dissimilarity on the getting surrender. This closures in reject of the vainglo- rious records data for re-transmission [14].

b) Foul play: A assigned in [14], wrongdoing is one reg- ular crash based thoroughly strike. It can likewise be re- ferred to as crumple principally based attacks.

c) power Fatigue: This sort of DoS attack birthplaces shockingly exorbitant development in a station making its availability extremely limited to the hubs. Such a di- version inside the station is because of a major amount of requests $(\mathrm{R}-\mathrm{S})$ and communicates over a station.

d) 4 Denial of Service assault at the group layer:

The vital element of the system layer of RSN is over- powering. The specific DoS ambushes charming zone in level are:

a) Ridiculing, repeating and confusion of site guests.

b) Hi flood strike: These attacks sources over the top guests in stations with the guide of blocking the channel with a shockingly unreasonable wide assortment of vain messages. Here a solitary pernicious hub sends a futile message which is then replayed by methods for the as- sailant to make an exorbitant site guests.

c) Homing: in the event of homing ambush, a hunt is made in the site guests for group heads and key adminis- trators which have the ability to close down the entire system. d) Specific sending: As the call recommends, in particu- lar sending, a bargained hub best sends a settled on cou- ple of hubs rather than every one of the hubs. This choice of the hubs is done based on the prerequisite of the aggressor to get his malevolent objective and thus such hubs does now not forward bundles of information.

e) Sybil: In a Sybil ambush, the aggressor duplicates an hub and gives it with in excess of one personalities to the contrary hubs.

f) Wormhole: This DoS assault causes migration of bits of information from its true capacity inside the group. Thiers area of information parcel is performed through burrowing of bits of information over a connection of low idleness.

g) Affirmation floodi:we are required at occasions in sensor systems while directing calculations are utilized. In this DoS attack, a pernicious hubs poofs the Ac- knowledgments providing false insights to the ordained nearhubs.

\section{SECURITY ISSUES AND PRIVACY CONCERNS IN WSN}
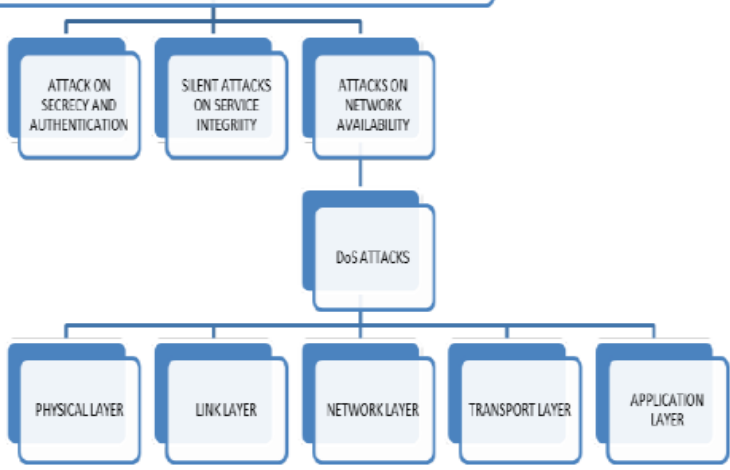

Fig. 1: Hierarchical Diagram of Security in Wireless Sensor Network.

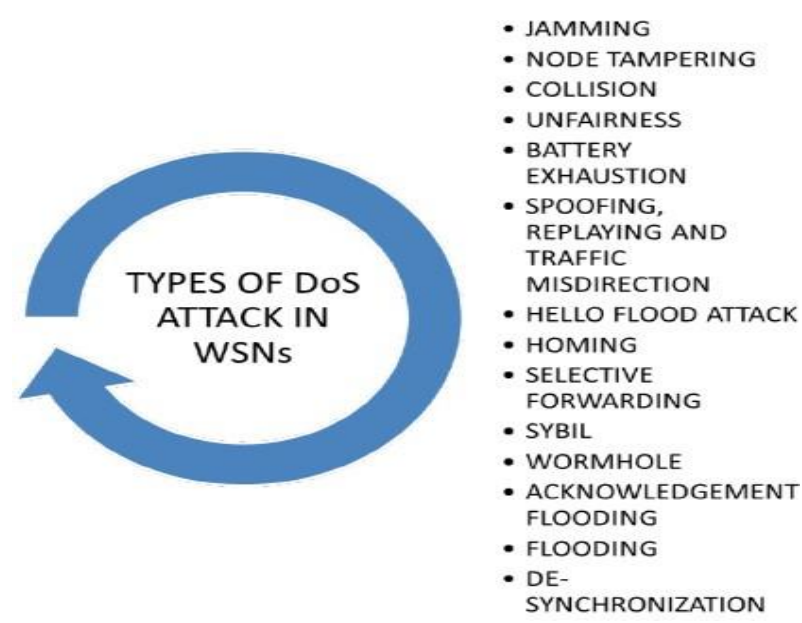

Fig. 2: Types of Denial of Attack in Wireless Sensor Network.

Denial of Service attack on the delivery lev- el:

This level of the RSN architecture gives correct of sta- tistics sending and avoids collision on account of high transaction in the hub. The Denali of Service attacks in this level given below:

a. Overflowing: allows to arranged clog of conversa- tion barrier through transfer of needless messages over the top visitors.Ii. Desynchronize: In de-synchronize assault, counterfeit messages are made at one or the two end points asking for retransmissions for cure of non- existent goof. These results in loss of imperativeness in one or both the end-factors in sporting out mock sum- mons.

Denial of Service assault at the product lev-el:

An utility layer of RSN completes the commitment of site guests administration. In furthermore act in light of the fact that the organization of software program for one-of-a-kind packages which consists of available the interpretation of records addicted to a understandable shape or enables in series of statistics by guiding probes 
[14]. In this level, a route-based totally DoS violence is originated by means of thought-provoking the sensor nodes to generate a big movement inside the direction in the direction of the source position [13] [14]. Figure two indicates all of above stated Denial of $S$ as saults in ex- traordinary layers of a ad-hoc sensor communicate. Sev- eral extra DoS assaults follow [7] [14] [15], [15]:

a) Relinquishment an Voracity Violence

b) Round of questioning

c) Darken Hovels

d) Hub Treason. Hub blame

e) Hub Oulabele

f) Inactive Info Assembly

g) Manufactured Nodule

h) Data Exploitation

Few of other protection \& privateness troubles in RSN are [7] [9] [10]:

a) Information Concealment

b) Information Veracity

c) Information Confirmation

d) Information Cleanness

e) Attainable quality

f) It Self-Organization

g) Salable Management

h) Safe Localization

i) Tractability

j) Strength and ability

As indicated by [14], the dangers approaching over RSN can similarly be named takes after:

a) Outside instead of in-house ambushes

b) Reflexive rather than energetic ambushes

c) Bit style against workstation greatness episodes As per [12], the assaults on RSN might be ordered as

d) Disturbance

e) Intervention

f) Amendment

g) Construction

The assaults on RSN can additionally named as:

a) Host-based assaults

b) System based absolutely assaults.

Security issues in MFID

Age In condition to IOT, MFID innovation is particular- ly utilized as MFID labels for programmed change of in- formation with no guide submersion. Yet, the MFID la- bels are helpless to various attacks from open air as a re- sult of the defective security notoriety of the MFID pe- riod. The 4 greatest ordinary types assaults and assur- ance inconveniences of MFID labels ([15] [15]) stand uncovered in Figure III which can be as per the follow- ing:

a) approved label incapacitating (wound on genuineness): The DoS attacks inside the MFID time prompts deple- tion of the MFID labels rapidly or Ever lastingly. that at- tack reduce a MFID label to breakdown and resist un- derneath the examination of a label reader, EPC giving trickiness towards a particular factual total character al- located for it. These DoS ambushes can be done at all, enabling the aggressor to impact the label exercises from one remoteness.

b) Unapproved label replicating (Attack on honesty): it taking pictures of documentation facts (like its EPC)esp. Thru the influence of the labels via scoundrel bibliophiles falls underneath a class. When the distin- guishing proof records of a label is dealt, replication of the label (cloning) is influenced attainable which to can be utilized to pass phony safety features in adding to awarding new liabilities in any venture utilizing MFID markers automated confirmation stages [15].

c) Unapproved label following (Attack on confidentiali- ty): A label might be followed through rogue readers, which may likewise bring about surrendering of sensi- tive information ike somebody's address. Hence from aconsumer's perspective, purchasing an item having a MFID label guarantees them no secrecy concerning the obtaining in their pursuit and really imperils their sup- pression. d) Replay strikes (Round on accessibility): Its kind dis- guise ambushes an attacker makes utilization of one la- bel's reaction to one rebel per users dare to parody the label[15].In replay assaults, Teaming up sign among the reader and the label is redirected, recorded and empha- sized upon the receipt of any inquiry from the per user at a later time, consequently reproducing the supply of the label. Other than this class, some extraordinary assur- ance susceptibilities of MFID innovation are [14]:

a) Figuring out

b) Power Analysis

c) Listening in

d) Man-in-the-center assault

e) Refusal of Service $(\operatorname{RoS})$

f) Satirizing

g) Infection

h) Following i.MurderingLabelApproach

\section{SECURITY ISSUES IN RFID}

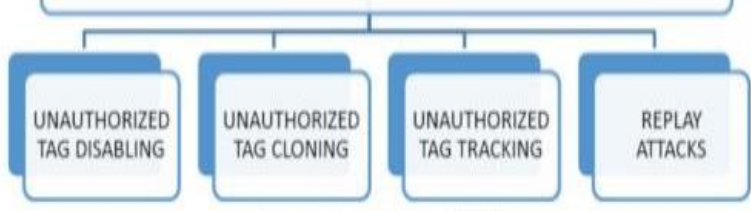

Fig. 3: Security Issues in RFID.

Sanctuary problems in health-associated tools constructed up the idea of IOT: Improvements conjunction of manufacturing with ecol- ogy has cemented the manner for immersed fitness ob- serving gadgets which could continuously circulation and proportion the data receive the sensor to the well- ness show along with various gadgets and social group over the web (The execution of social network with the sensor certainties can be seen in [13], [10] and [11]). The execution of automated gathering of certainties by methods for the sensors and bringing in it to the differ- ing open systems through a web server reports some over the top lack of protection inside the entire records communicate procedure from screen to the Internet. The possibility in its objective apparatus (FITBIT), the creators of ([13], [12]) have analyzed the accompanying as the guideline security presentation way such wellbe- ing checking gadgets occupied in uniting with Internet:

a) Strong content login data: Throughout login the rec- ord associated with wellbeing watching gadget, the true blue secret key of the buyer is identified in the webserv- er in clear content that is then confirmed in log reports.

b) Solid substance HTTP records setting up: The sensor information is sent to the net servers as fundamental HTTP orders with no extra security or encryption. Such exposed HTTP summons can be effortlessly involved for drawing nearer to various features of a customer ac- count identified with the prosperity watching contrap- tion. From the above communicated susceptibilities it's far clear that the safety efforts associated in the prosperi- ty related development which are socially related over the web do not have the best possible trials to adapt to all the privateness issues of the stop clients and spots the clients in danger of exposing treasured records about their wellbeing to unknown employees with evil plans. In view of the above-alluded to assurance flaws, numer- ous other wellbeing and security inconveniences existent them selves in the teach of IOT. A two them:

a) Theft of infiltrating certainties identical budgetary foundation mystery code.

b) Simple agreeability to private information likes advo- cate manage, advocate wide assortment et cetera.

c) It might moreover bring about open get admission to private data like monetary popularity of an association 
d) An attack on any individual instrument may likewise bargain the integrity of the various related gadgets. In this manner the interconnectivity has a major downside as an unsafety disappointment can disturb an total group of gadgets.

e) Dependence on the Internet marks the entire IOT en- gineering helpless against infection strike, worm attack and the vast majority of the other security dis advanla- beles that comes with any Internet connected processing gadget et cetera.

\section{Conclusion}

In this paper we've studied the majority of the security flaw scurrent in the Internet of Things which can turn out to be very damaging in the advancement and usage of IOT in the one of a thoughtful fields. So reception of comprehensive safety measures ([13] [14] [14] [15]) mention in the above targeted wellbeing blemish and in addition usage of diverse intrusion identification frame- works ([11] [15]), cryptographic and stenographic high- lights ([5]) in fact salternate methodology are utilizing of proficient strategies for communique ([13]) will real- ize a effecitve prominent agreeable tough IOT founda- tion. In end, all might want to in dicatethat additional exertion on change of secured count for the current IOT foundation before go for similarly improvement of most recent execution methodologies of IOT in every day life may show to be an additional productive and systematic method.

\section{References}

[1] AashimaSingla, RatikaSachdeva, "Review on Security Is- sues and Attacks in Wireless SensorNetworks" IJARCSSE Volume3, Issue4, Apr 1123ISSN: 1107 114X.

[2] Sen,Jaydip. "Security and privacy challenges in cognitive wireless sensor networks arXiv: 1302.1310 (1123).

[3] Bianchi, "A comparative study of the varioussecurity ap- proaches used in wireless sensor networks,'IJAST, vol.13, (1120) Apr, pp. 1510.

[4] T. A. Zia, "A Security Framework for Wireless SensorNet- works", Bhattasali, Tapalina, RituparnaChaki, and SugataSanyal. "Sleep Depri- vation Attack Detection in Wireless Sensor Network."

[5] Roy, Bibhash, Suman Banik, ParthiDey, Sugata Sanyaland Na- benduChaki, "Ant colony based routing formobile ad-hoc networks towards improved quality ofservices." JETCIS 3.1 (1122): 10-14.

[6] M. Saxena, "Security in Wireless Sensor Networks-ALayer based classification", Technical Information, Centerfor Education and Research in Information Assurance\& Security-CERIAS, Purdue University.

[7] B. T. Wang and H. Schulzrinne, "An IP traceback instrument for in tel- ligent DoS assaults",ECE Meeting vol. 2, (1110) May 2-5, pp. 901-904.

[8] Ahmad Abed Alhameed Alkhatib, and Gurvinder Singh Baicher. "Re- mote sensor organize design." International gathering on PC systems and correspondence frameworks (CNCS 1122).

[9] Al-Sakib Khan Pathan, "Refusal of Service in Wireless Sensor Networks: Issues and Challenges", ACMR, Vol. 6 (Edited by Anthony V. Stavros), ISBN: 978-1-10876-106-8, NSPI., USA, 1120. [14 Sunil Ghild- iyal, Amit Kumar Mishra, Ashish Gupta, Neha Garg, "Examination of Denial of Service (DoS) Attacks in Wireless Sensor Networks" IJRET: UJRET; eISSN: 1123-1115 | pISSN: 1123-7308

[10] Khoo, Benjamin. "MFID as an empowering influence of the web of things: issues of security and protection." IOT 1121 International Confer- ence on and fourth International Conference on Cyber, Physical and Social Computing. IEEE, 1121.

[11] Burmester, Mike,and BrenoDe Medeiros. "MFID security: assaults, countermeasures and challenges." The fifth MFID Academic Convocation, The MFID Journal Conference. 1110.

[12] C. Karlof and D. Wagner, "Secure Routing in Sensor Networks: Attacks and Countermeasures", Elsevier's ANJ, Special Issue on SNPA, (1110) September, pp. 112-110.

[13] Zhou, Wei, and Selwyn Piramuthu. "Security/protection of wearable wellness following IOT gadgets."(CISTI), 1124 ninth Iberian Conference on. IEEE, 1124.

[14] Aggarwal, Charu bdelzaher. "Coordinating sensors and informal organizations." Social System Data Analytics. Springer US, 1121. 110122. 